\title{
THYROTOXICOSIS
}

\author{
By Sir Carrick Robertson, F.R.C.S., F.R.A.C.S., Hon. F.A.C.S. \\ - Auckland, Nerv Zealand
}

A goitre is an enlargement of the thyroid gland. It may be either a diffuse smooth enlargement or a lumpy one. The lumps are due to the presence of nodules, varying in size from a pea to a cricket ball; this type used to be called adenomatous goitre, but the pathologists say these nodules are formed by alternating phases of hyperplasia and involution (Reinhoff \& Lewis) and are not true adenomata, so it is better to reserve the name ' adenoma' for the single adenoma which may occur in the thyroid as in any other glandular organ. Both the diffuse and the nodular enlargements can be present without doing apparent harm to the patient : but as time goes on, something happens to the secretion of the thyroid hormone and an insidious change takes place in the health of the patient, which we call thyrotoxicosis. These goitre enlargements are prone to occur in parts of a country where there is an absence of iodine in the soil. Without iodine, the synthesis of thyroid hormone or thyroxin, cannot be completed.

The function of this little gland is to trap the inorganic iodine in the blood as it passes through and use it in the manufacture of thyroxin. In spite of its small size, it contains over 20 per cent. of all the iodine in the body. It is supplied by four comparatively large arteries so that all the blood in the body must pass through it many times a day.

Thyroid hormone stimulates the metabolism of the body and it becomes of great importance at puberty, so that children living on an iodine deficient soil are very prone to have diffuse enlargements of the thyroid gland. It is almost as if the enlargement of the thyroid at puberty may represent an addition in the size of the factory, in order to augment its efficiency in abstracting and utilizing more and more of the iodine present in the blood. It is in childhood that nearly all nodular goitres commence their career : for if the diffuse enlargement persists till about 20 years of age, small nodules begin to form in the substance of the gland. Once this has happened, it is unusual for them ever to disappear. On the other hand, administration of quite small doses of iodine or thyroid extract before nodulation occurs, is usually a preventive of further goitre trouble.

The quantity of thyroid hormone in the blood stream is controlled by the anterior pituitary gland : should there be too little, a thyroid stimu- lating hormone (T.S.H.) goes forth from the pituitary which causes increased activity in the thyroid and a rise in the production of the thyroxin. The opposite effect takes place if there is not sufficient thyroxin in the blood stream.

A few years ago, it was discovered that the production of thyroxin by the thyroid gland could be suspended by administering thiourea, thiouracil or other thio compounds by the mouth. These drugs act by their toxic effect on the thyroid gland itself : they render it incapable of synthesizing the blood iodine into thyroxin. Continued administration of the drug eventually leads to a state of myxoedema.

It should be remembered that thiouracil has no action on the anterior pituitary gland, so that with the decreased production of thyroxin, there is a reflex increase in the quantity of T.S.H., the stimulator of cellular activity in the thyroid. A microscopic section of a gland under treatment with thiouracil shows a hyperplasia similar to that seen in Graves' disease, but all to no avail, the thiouracil inhibits the formation of thyroid hormone in spite of the activity in the acini of the gland.

It is useful to have a working classification of pathological enlargements of the thyroid. One in general use is :-

(I) Nodular or lumpy enlargements :
(a) Simple

(b) Toxic

(2) Diffuse or smooth enlargements :

(a) Simple, such as colloid goitre in the young.

(b) Toxic or Graves' disease.

(3) Inflammatory - thyroiditis, abscess, gumma, Riedel's woody thyroid.

(4) New growth-benign or malignant, cysts, hydatids, dermoids.

(5) Hashimoto's disease-a rare condition in which the tissue is infiltrated with lymphocytes. For full information see article by Joll. In this paper we are only concerned with thyrotoxicosis as occurring in the toxic nodular and toxic diffuse groups.

\section{Nodular Goitres}

A patient with a nodular goitre need not necessarily have a visible tumour in the neck-the nodules may be small and deeply situated. The size of a 
puberty goitre often decreases, even without treatment : possibly the extra fat and development of the neck muscles, as the patient grows older, covers any swelling that may remain, so that even if there was a recognizable swelling as a child, it is forgotten. Later in life, pregnancy, general infection or some serious emotional disturbance, such as domestic unhappiness 'occurs, and the simple gland becomes a toxic one. The nodules increase in size and the goitre again becomes obvious.

During acute systemic infections, or even during chronic infection elsewhere in the body, the thyroid gland, especially the already diseased one, (whether diffuse or nodular) may become inflamed. As a result, a severe thyrotoxicosis can develop. There may still be people who advocate removal of septic tonsils for thyrotoxicosis-it used to be a very general treatment-but it fell into disrepute because the thyrotoxic symptoms once started, are not appeased by taking out the septic focus. However, as a prophylactic measure, it is sound procedure.

\section{Diffuse Goitres}

The other common form of toxic goitre is primary Graves' disease. This may appear at almost any age and, in what up till then, has been a perfectly healthy gland. The same causes that turn the simple nodular into a toxic nodular goitre are probably present. The symptoms are so similar that no useful purpose is served by differentiating them.

A microscopic section of a toxic diffuse goitre shows great increase in the size and activity of the cells lining the vesicles throughout the gland. Exactly the same hyperplasia is seen in toxic nodular goitre except that it does not occur throughout, but only in patches.

\section{Diagnosis of Thyrotoxicosis}

When the signs are well developed, there can be no difficulty. The combination of staring eyes, nervous restless movements, tachycardia or shortness of breath with a visible almost pulsating goitre, can hardly be mistaken. In such a case, a B.M.R. is not necessary for the diagnosis, but may be useful if taken from time to time in checking the progress of whatever treatment is adopted.

However, in the early stages, or milder cases, diagnosis may be anything but easy; for in such a case, the following symptoms will be found :-

(I) The predominant symptom of all cases is 'tiredness' and this in spite of the fact that they feel full of energy and can often do a full day's work.

(2) Shortness of breath on exertion and tachycardia.
(3) All kinds of psychotic symptomsemotional, depressive, irritability and phobias of various kinds.

It is obvious that there are many causes of such symptoms, for example, the anaemias, early phthisis, anxiety neurosis. The first can be ruled out by obtaining a blood count, the second by an $\mathrm{X}$-ray of the chest. A former H.S. of mine who is now an authority on chest diseases, told me that when he first went into the wards of the Brompton Hospital for Diseases of the Chest, he thought all the patients looked like early Graves' disease.

There are some further points in the signs and symptoms of thyrotoxicosis which should be mentioned before discussing differential diagnosis, especially the difficult problem of distinguishing between mild thyrotoxicosis and anxiety states.

$A$ palpable thyroid gland : It is quite unusual to find a normal sized thyroid in a thyrotoxic patient, even in primary Graves' disease, which attacks an apparently normal gland; the sequent hyperplasia makes the gland larger. A normal thyroid, except for the isthmus, cannot be felt except in a very thin neck, so if the gland is palpable, it is probably enlarged. A useful test is to ask the patient to stretch his or her neck and swallow: this will sometimes induce a hidden swelling in the lower poles to come out from behind the sternum, because all thyroid swellings move up and down during deglutition. This sign is also useful in distinguishing a large nodule in the upper pole from a swollen lymphatic gland or other tumour of the neck.

In doubtful cases, it is helpful if the patient admits to a goitre during adolescence, because this greatly increases the chances of there still being some enlargement remaining.

Tachycardia which does not settle after a short rest. The heart sounds will be found to be loud and slapping. When asked if they have any discomfort in bed at night, they will frequently complain of a 'bumping of the heart.'

$A$ fine tremor on holding out the hands, difficult to describe and of unknown cause, but very characteristic.

Eye signs : This will vary from a staring appearance to marked exophthalmos, usually in both eyes, but may be more marked on one side than the other.

Lid Lag (von Graefe) : This is a very distinctive and helpful sign. On following an object downwards, the lid moves more slowly than the eyeball and a momentary glimpse of the white sclerotic between the cornea and lid is obtained. This is due to a spasm of the eyelid and not to exophthalmos (Joll).

Other symptoms : Flushes, especially of the neck and upper chest, free perspiration, clammy hands. 
Insensitivity to cold, loss of weight and yet good appetite. Weakness of the quadriceps femoris, shown by difficulty in walking upstairs.

The basal metabolic rate will be raised.

\section{Differential Diagnosis}

Anxiety state in a young woman with a slightly enlarged thyroid is a difficult problem, not always helped by a B.M.R. test, because the anxious patient finds it hard to breathe quietly during the test. Their fear makes them nervous, tremulous and inclined to sweat, but these symptoms are not associated with increase in appetite, loss of weight or increased tolerance for cold : they may even have cold blue hands. While talking to you, they take deep sighing respirations. Tachycardia differs in that it does not persist when the patient is lying comfortably in - bed - they never complain of heart 'thumping' when resting. The pulse pressure in thyrotoxicosis is commonly found to be raised but not in anxiety states. I believe this test to be more valuable than a B.M.R. in this type of case.

(I) Microcytic anaemia with Plummer Vinson syndrome : The major complaint will be difficulty in swallowing, but if the patient has a goitre, she may regard it as the cause. The anaemia will have made her tired and rather short of breath, so that there is more than a semblance of toxic thyroid. However, so seldom does real difficulty in swallowing occur from enlarged thyroid alone, that other causes of dysphagia should be sought. In my practice, I have often seen this association of goitre and Plummer Vinson syndrome, so there may be some connection between the two.

(2) Hypothyroidism: Although a patient may have some thyroid enlargement, feel tired and become easily out of breath, these symptoms can be due to a hypothyroid state. Their general appearance, loss of eyebrows, puffy hands and lack of nervous energy, with forgetfulness and headache, should lead to the correct appraisement.

(3) Remissions : The fact that there are remissions in Graves' disease is troublesome, for the patient may arrive for consultation in one of her good weeks, and yet only the week previously she may have been showing obvious signs of a severe thyrotoxicosis. A therapeutic iodine test is often valuable, for if ten drops of Lugols solution given t.d.s. for a week gives marked relief, the cause is surely one of thyrotoxicosis. Thiouracil has also been used as a therapeutic test, but its drawbacks are that it has to be given for some weeks before there is clinical improvement.
It is well to remember that the patient before coming to the consultant, may have recently been prescribed iodine ; without the knowledge of this, it may be difficult to find enough evidence on which to base the diagnosis. It is best to cultivate the habit of always asking a goitre patient for her iodine history. Some patients with a fairly high degree of thyrotoxicosis have been found taking Lugols m. x, t.d.s. for a year and in one case, for nearly three years. These patients make light of their continuous tachycardia and exhaustion after slight exercise, for their faith in the treatment is such that they have developed a psychological mask, difficult for the examining doctor to penetrate. It must be remembered that iodine does not cure thyrotoxicosis. Mellanby says, "It is undoubted that during the administration of iodine, the patient feels better and the basal metabolic rate is lowered and remains lower while the iodine is continued, although not so low as in the early days of treatment.'

There is never any need for hurry in advising a patient with a doubtful thyrotoxicosis to have an operation-no harm comes to them by waiting for a clear indication, whereas, removal of thyroid tissue in a patient whose symptcms are due, say, to anxiety neurosis, only adds hypothyroidism to her troubles.

Treatment of thyrotoxicosis is either by one of the thio compounds or by partial thyroidectomy. They both reduce the production of thyroxin or thyroid hormone. In surgery, this is effected by reducing the size of the factory, while thiouracil inhibits the synthesis of thyroxin by the thyroid gland and in spite of increased activity of the acinar cells caused by pituitary stimulation-in other words, the factory is enlarged, but the output ialls.

Thiouracil treatment: This has to be carried out for long periods. The advantage is that, except in severe cases, this treatment may be carried out while the patient continues with her normal work, but it has to be carried out under supervision for long periods. The dose of the drug should not be more than 0.2 gms. t.d.s. During the whole time thiouracil is being taken the patient must be under supervision, for it is a toxic drug and about 20 per cent. of cases get toxic rash, enlarged glands, pyrexia, or a feeling of depression, but the most important toxic effect is on the white corpuscles in the blood, so that an estimation of the leucocytes should be made at least weekly. At the end of the first week of treatment, there is usually a fall in the number of leucocytes. If it is not less than 4,000 and the polymorphs are still in excess of the lymphocytes, there is no need to stop the treatment until the next count is taken, when very often the number of leucocytes has risen to the normal number. 
I have seen a case which had been treated for just about a month without any blood counts. She became very ill and was sent into hospital where we found she had not a single white cell in the blood counting chamber, She very quickly died, in spite of anything we could do for her.

In a suitable case, there is usually a very great improvement at the end of a few months, and at the end of six or seven months, the patient thinks she is completely cured, but if the drug is then stopped, there is very likely to be a relapse, so that a continuance of the drug in small doses, say, .05 gm. per day, has to be carried out for a long time, perhaps a year, or even two. It seems that treatment for such a long time may not be entirely without danger, for in rats at least, Purvis and Griesbach have produced adenocarcinoma of the thyroid in 50 per cent. of the experimental animals by administration of thiourea for a period of two years. It is only fair to state that we have no evidence of thyroid carcinoma in man which could be attributed to this treatment.

As against this long period of treatment, thyroidectomy usually gives full recovery in three or four weeks, and if it is performed in a good goitre clinic, mortality is low, under $\frac{1}{2}$ per cent. When thiouracil was first introduced, we treated 45 cases of all types of thyrotoxicosis with this drug and at the end of eighteen months we were able to say that 50 per cent. had done very well. Since that date, however, six of these relapsed and so the percentage of recovery in these original cases is now only 35 per cent. It would seem that a selection of cases for this treatment is important. Can we say which patient with thyrotoxicosis will be relieved by this drug?

First of all, I would put the young patient with a smooth goitre - and a small one at that. Many of these have done very well, but there are certain reservations, which will be elaborated later when we are dealing with toxic nodular goitre.

Secondly, the elderly thyrotoxic patient of 60 or 70 , whose heart and kidneys have become damaged owing to the length of time during which the toxin has been circulating in theirblood, makes the surgical risk greater.

Thirdly, those cases which have already had thyroidectomy and have recurred. It is well known, that in this recurrent type, the dangers of a surgical operation are greater than when an operation is done for the first time. There is a much greater risk of injury of the recurrent laryngeal nerve and of the parathyroid glands. These cases often respond quite quickly to small doses of thio, say .2 gm. daily, but we have had several failures, because the swelling of the remnants has been increased by administration of the drug and caused a choking feeling.
In the operation of thyroidectomy as we do it, a very small piece of the posterior part of the gland is left lying closely along each side of the postero-lateral aspect of the trachea.

Fourthly, it has been strongly advocated, especially by the Lahey Clinic, as pre-operative treatment in cases of severe thyrotoxicosis. One of the thio compounds is given, until the B.M.R. has come down to 0 . After that, at least two weeks treatment must be given with iodine, so that altogether, two or three months may elapse before the operation is performed. The iodine is necessary because there is no doubt that a thiouracilprepared case is more difficult to operate upon than one which has been prepared with iodine. The use of iodine makes the gland less vascular and so the operation is easier.

It is less difficult to be certain about contra-indications. First, those who cannot carry on the treatment because of toxic reaction such as leukopenia. Secondly, nodular goitre. It has been pointed out previously in this article that the administration of thio drugs causes an increase in the hyperplasia of the thyroid gland. This means that the volume of the gland is increased. In many cases of nodular goitre, though not all, the trachea is compressed or displaced, so that some interference of thee airway is experienced. In an extreme case, there is a whistling inspiration due to the narrowing of the trachea and the patient is very short of breath in fact, she may be regarded as a thyrotoxic when? she has no other reason for shortness of breath than the insufficient capacity of the trachea. Such extreme cases are rare, but it is not uncommon to see, at an operation on a nodular goitre, indentation and softening of the trachea as a result of the pressure. The patient may have complained of no symptoms of this-for so slow and insidious is its development that the patient adjusts herself to it, and is unaware that she has any obstruction to her airway. These are easy to see at operations, but there is another type, not so easily recognized, where a small nodule in the posterior part of the gland has insinuated itself between the spinal vertebrae and the oesophageal-tracheal tract. When such a nodule is found, you may notice as it is withdrawn that it is shiny, glistening and of a greyish colour, like a waterworn stone. The appearance vanishes very quickly, once it has been contaminated with the blood and serum of the wound. It is probable that the up and down movement of the trachea during swallowing has caused a thickening of the capsule of the nodule, thus altering its external appearance from the rest of the gland. In these cases, there will be no indentation of the trachea, but it must surely cause discomfort such as a tight feeling in the neck. Several such cases have been found in cases 


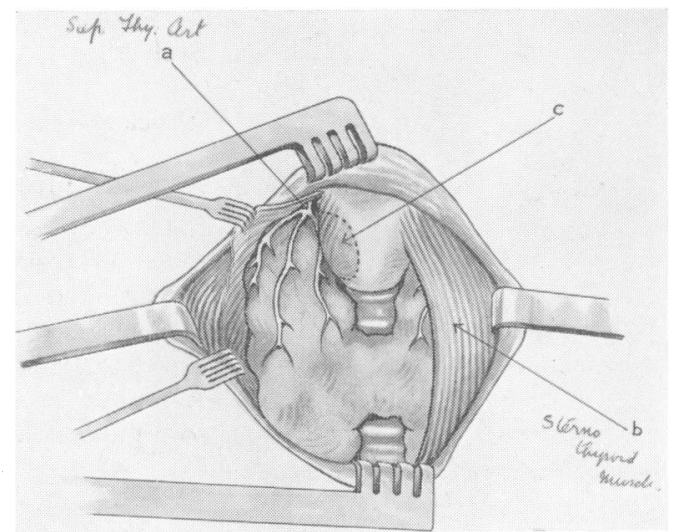

FIG. 1.- $a$. Sup. thy. art. breaking up into three branches. $b$. The upper pole covered by the sternothyroid muscle. Until this is retracted or divided the arcery is not seen sufficiently. c. Nodule behind the larynx.

treated with thiouracil who refused to carry on because they felt uncomfortable especially noticeable whrn swallowing.

A similar experience has been found in operating on primary Graves' disease, which had failed with thiouracil treatment. In these cases, no nodule will be found behind the trachea, for nodular formation has not begun: however, the upper pole will be found to have insinuated itself between the larynx and the spine. Practically all cases of Graves' disease have an enlargement of the upper pole, and in very many of them, part of the pole will be found behind the larynx. In one case, a tiny early nodule was found in this retro-laryngeal portion as shown in the photograph and drawing. The administration of thio will increase the size of the gland, including the part behind the larynx, and make the patient conscious of discomfort such as a slightly constricted feeling in the neck or some alteration in swallowing. Although their B.M.R. had become normal, or nearly so, at first one thought these patients were not making sufficient effort or were neurotic. That this was not the case is shown by their complete recovery after thyroidectomy. Another reason for advocating operation in a toxic nodular goitre is that only very seldom have we seen nodules disappear after the treatment. They usually remain, and therefore must be a latent source of a recrudescence of the disease, or they may eventually become malignant.

Thirdly, all patients who live too far away from centres where blood counts and B.M.R.s can be done.

Fourthly, a patient whose heart symptoms are predominant. The first symptoms to clear up on thiouracil are the flushes and the nervous excitement : tachycardia last of all. It may take several months before the tachycardia is relieved. Although there are cases of fibrillation which have got better on this treatment, there is no doubt in our mind that surgery is more suitable and can be relied upon to cure the tachycardia and the fibrillation with more certainty and in a much shorter time.

A further contra-indication is exophthalmos. Under thiouracil the exophthalmos may increase although the other toxic symptoms abate. Dobyns of the Mayo Clinic believes that the thyroid stimulating hormone of the pituitary produces exophthalmos owing to alteration in fat metabolism and fibrous tissue reaction. Thus many of the changes in the orbital tissue which produce exophthalmos are expressions of generalized tissue changes (Dobyns).

\section{Pre-operative Treatment}

The preparation of mild cases consists of giving them iodine (Lugols, m. $x$ t.d.s.) for two weeks before the operation. They need not come into hospital. In the severe cases, patients are taken

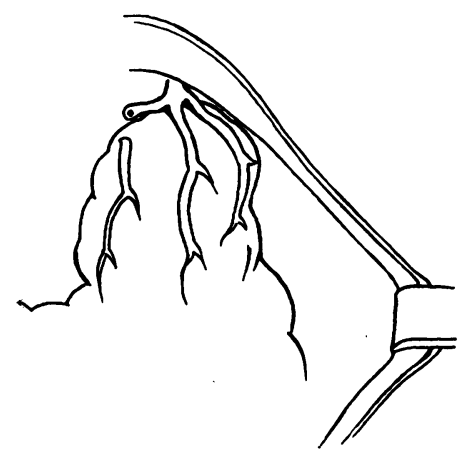

1

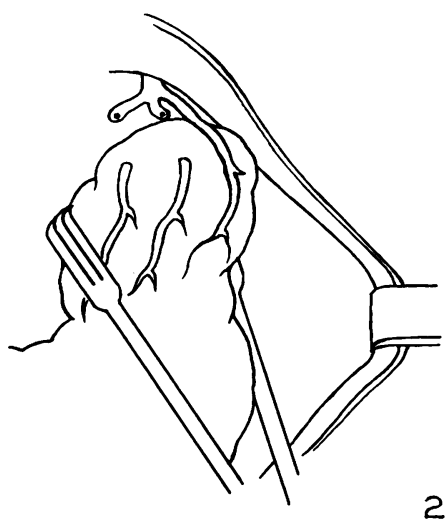

2

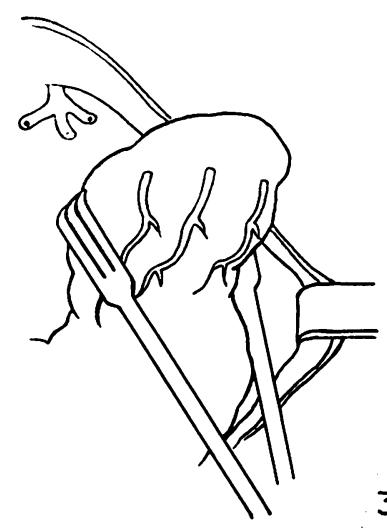

FIG. 2.-Three stages in severing the branches of the superior thyroid artery. 


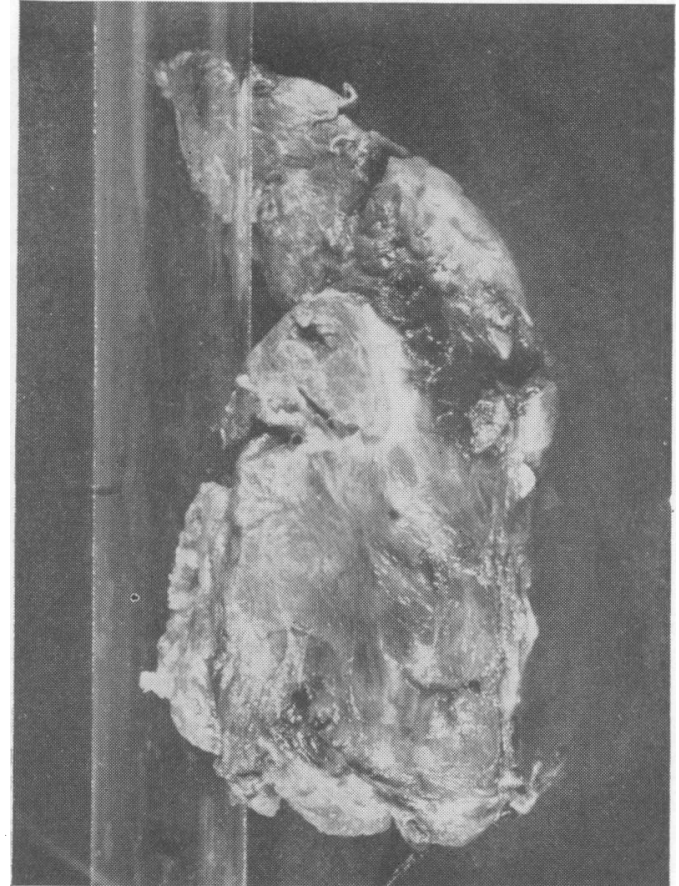

FIG. 3.-Left lobe of thyroid removed at operation. A glass tube replaces the trachea and larynx. This illustrates the fold of the upper pole lying behind the larynx.

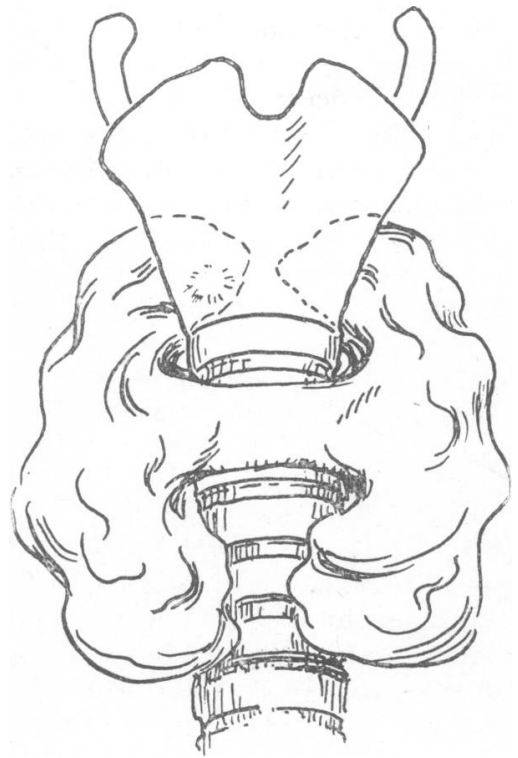

Fig. 4.-A line drawing made in explanation of the photograph.

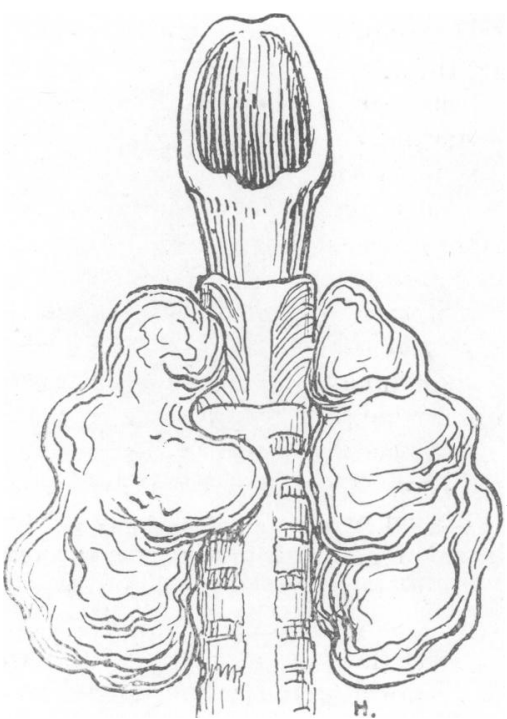

Fig. 5.-To show a glistening nodule behind the trachea. This obviously cannot be seen from the anterior aspect.

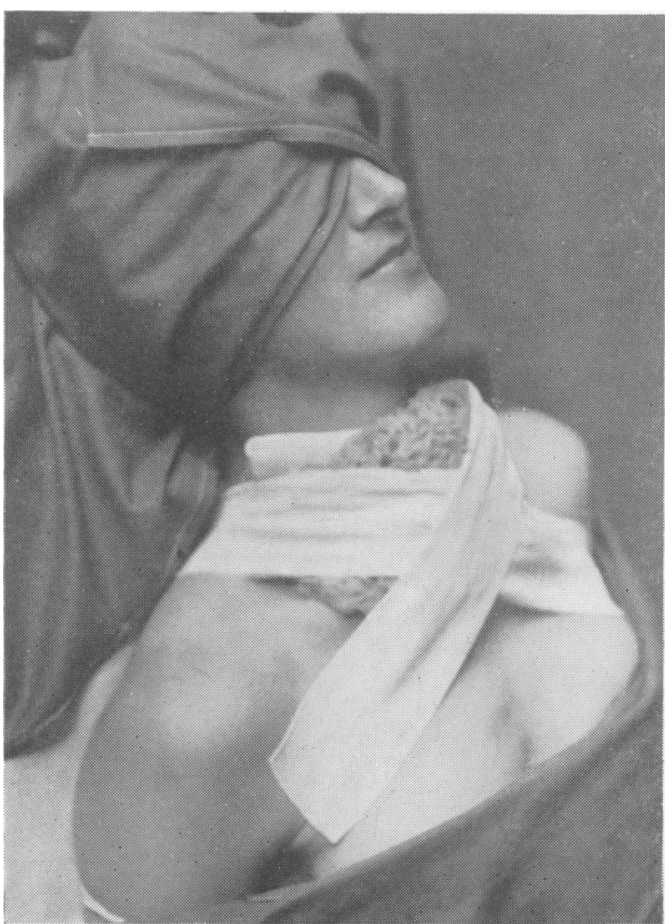

FIG. 6.-Post-operative dressing. 
into hospital after a few days at home on iodine and rest. In hospital, they are put to bed, the iodine continued, sedatives administered freely if necessary, and they are encouraged to drink large quantities of water. Patients with thyrotoxicosis use up more Vitamin $\mathrm{BI}_{\mathrm{I}}$ than normal-the amount of this vitamin contained in the daily food is not enough, and much benefit accrues when they are given large doses of Vitamin $\mathrm{BI}_{\mathrm{I}}$. During the time they are waiting a daily dose of $50 \mathrm{mgm}$. of thiamin chloride is given intravenously. Added to this, we give them oxygen to breathe for half an hour, two or three times a day. This was originally started to enable patients to get used to the B.L.B. mask, or a catheter through the nose, for the administration of oxygen after the operation. It was found that practising breathing oxygen before the operation gave the heart considerable relief and we now use it as a pre-operative routine. Most patients are ready for operation in a week. It is rare that they need 10 days of this treatment.

On the morning of the operation heavy premedication is given. On arrival in the theatre, they are given a local analgesic in the neck and a light oxygen and ether anaesthesia.

\section{Details of Operation}

Position: A small pillow under the shoulders to extend the neck.

The incision: This is made about two fingers breadth above the manumbrium, reaching from onc external jugular vein to the other and made in 'the line of cleavage' of the skin. When the goitre is large, it is better to make the incision rather higher, as the skin flaps tend to sink and the final scar may be just above the sternum or even on it. 'The higher scar is less noticeable.

'The skin, superficial fascia and platysma are incised and the upper and lower flaps thus made are dissected up and down, respectively, displaying the deep fascia. The special goitre retractor is now inserted.

A longitudinal incision is now made in the midline through the cervical fascia. The left sternohyoid muscle is retracted laterally and the left sterno-thyroid divided between forceps. This will expose the left lobe except the summit of the upper pole. A pyramidal lobe is sometimes present and should be completely removed at this stage as a recurrence makes an unsightly reminder of an operation that has not been sufficiently thorough.

The so-called suspensory ligament of the thyroid is divided as this enables the upper pole of the gland to be pulled down slightly. Haemostatic forceps seize the visible anterior branch of the superior thyroid artery, which is divided between them; giving a little more freedom to pull the upper pole downwards, exposing one after another, the three or four branches of the artery on the lateral and posterior surfaces. These are caught in forceps and divided as they appear.

Only areolar tissue and the blood vessels themselves are grasped. No thyroid tissue should be included.

The upper pole now peels out freely, all the guy ropes or branches of the artery holding it in position having been divided. When the pole is a high one, there is nearly always a piece of it curling under the thyroid cartilage. Its removal will be assisted by putting the forefinger of the right hand behind the gland and pushing the pole gently forward. It is very important to leave none of the upper pole : by this method it is easy both to see and make sure.

Attention can now be turned to the lower pole which is pulled up or out from behind the sternum with Lahey forceps. It is amazing to see how easy it is to enucleate even a very large substernal prolongation once the upper pole is free.

Release of lower pole: The inf. veins running from the lower pole into the mediastinum are clamped and divided. The trachea is not yet visible because it is covered with areolar tissue : this tissue is parted with scissors until the trachea is seen. The scissors are then passed up between the isthmus and the trachea, so as to protect the trachea when the isthmus is cut with a scalpel.

Release of left lobe: Two or three artery forceps now grip the gland tissue lying on the left side of the trachea, thus picking up invisible branches of the inf. thyroid artery as they pass into the hilum. 'The lobe is grasped in the fingers of the left hand and by further clamping and cutting from the midline outwards, $\frac{7}{8}$ of the gland is removed, the fingers feeling for deep or retrotracheal nodules.

$A$ branch of the inferior thyroid artery runs in a tiny groove of the gland between the upper pole and the isthmus, and must be carefully clamped before removal of the lobe. This artery lies near the site of entry of the recurrent laryngeal nerve into the cricothyroid membrane and if it slips out of the forceps, the nerve may be injured while diving for it. Should bleeding occur from there, it is better to ligate the main inf. thyroid artery as it emerges from under the carotid sheath, and before it turns up to spread over the gland. The artery forceps are now replaced by ligatures of linen thread.

Freeing and removal of right lobe: This is a similar operation to the left side but the sternothyroid rarely needs to be divided.

The stumps of the gland on the two sides are now inspected and if there is any oozing at all, two things may be done. The stump may be sutured with linen thread on a Mayo needle suturing the capsule on the outer side to the remains of 
the hilum on the trachea. Care must be taken that the needle does not go too far into the hilum as there is a risk of including the recurrent laryngeal nerve in the suture. If the oozing continues, the main inferior thyroid artery may be tied near the carotid sheath.

Closure of wound : The cut ends of the sternothyroid muscle are united with a ligature. The sides of the sternothyroid muscles are brought together over the trachea with a single stitch.

The median incision in the cervical fascia is closed by three separate sutures, picking up tissue outside the ant. jugular plexus to avoid the veins. The skin edges are then closed with a continuous horse hair suture and no drain is inserted, for if all the vessels are clamped before division, the wound will be bone dry.

The wound is now compressed with a marine sponge dressing and two strips of elastoplast as in diagram. This is left on for 48 hours and then this dressing and the skin sutures are removed.

In male patients, it is sometimes necessary to divide the sternohyoid and even part of the sternomastoid muscles to give a good exposure. When suturing these muscles at the end of operation, be sure to include in the suture the blood vessels which bleed from the cut ends.

\section{Post-operative Complications}

Haemorrhage : This usually occurs in the first few hours. It cannot be too strongly pointed out that if the bleeding occurs under the muscle layer, no swelling of the neck will be seen ; the only sign will be blue lips from cyanosis. Any suspicion of this warrants the immediate opening of the wound, and if clots are found they are cleared out and the wound packed until the next day. It is sometimes found on clearing out the clot that there is still cyanosis and some difficulty in breathing. It is due to a spasm of the vocal cords from irritation of the recurrent laryngeal nerves and should be treated by an immediate tracheotomy. The tracheotomy tube can be removed in a day or two when spasm goes and no evil effects in the wound have been noticed, and since the use of penicillin in the wound there has not even been undue delay in healing.

Tetany may occur. It is usually noticed about 48 hours after the operation. As soon as it is diagnosed we give an intravenous dose of calcium gluconate and continue for the next two or threc days to give calcium lactate by the mouth, together with Vit. D. in the form of cod liver oil. In nearly every case there has been no further trouble.

Difficulty in swallowing, especially in the swallowing of fluids, due to bruising or injury of the superior laryngeal nerve, so interfering with the sensation of the larynx that drops of fluid may go ' down the wrong way.' This clears up in a few days. In the meantime solid or semi-solid fogd is given without any trouble.

Loss of voice is due to bruising or injury to the recurrent laryngeal nerve, causing paralysis of a vocal cord, which may be permanent. If the paralysis does not appear for 48 hours or more, then it is probably due to pressure on the nerver from the general oedema and outpouring of serum in the wounded tissues. This type improves leaving no sign of cord paralysis. A patient with a permanent unilateral paralysis will whisper fo늠 two to three months. After that the active cord has learnt to cross the mid line during phonation and the speaking voice becomes almost normal

\section{BIBLIOGRAPHY}

REINHOFF and LEWIS (1928) Archives of Surg., 16, 79. JOLI, C. H. (1939), Brit. F. Surg. Oct., 27, $371-380$.

PURVIS, H. D. and GRIESBACH, W. E. (1947), Brit. F. Exp. Path. 28, 46.

LAHEY, F., ' $\Lambda$ nnals of the R.C.S.,' I, No. 6, 277.

DOBYNS (1946), Western Fournal of Surg., Obst. and Gyn. $54 .+1 \%$

\section{RUTHIN CASTLE, NORTH WALES}

A Clinic for the diagnosis and treatment of Internal Diseases (except Mental or Infectious Diseases). The Clinic is provided with a staff of doctors, technicians and nurses.

The surroundings are beautiful. The climate is mild. There is central heating throughout. The annual rainfall is 30.5 inches, that is, less than the average for England.

The Fees are inclusive and vary according to the room occupied.

For particulars apply to THE SECRETARY, Ruthin Castle, North Wales.

Tolograms : Castle, Ruchin.

Telephone: Ruehin \&s 